\title{
Self-similar solution of the unsteady flow in the stagnation point region of a rotating sphere with a magnetic field
}

\author{
H. S. Takhar, G. Nath
}

\begin{abstract}
The unsteady flow and heat transfer of a viscous incompressible electrically conducting fluid in the forward stagnation point region of a rotating sphere in the presence of a magnetic field are investigated in this study. The unsteadiness in the flow field is caused by the velocity at the edge of the boundary layer and the angular velocity of the rotating sphere, both varying continuously with time. The system of ordinary differential equations governing the flow is solved numerically. For some particular cases, an analytical solution is also obtained. It is found that the surface shear stresses in $x$ - and $y$-directions and the surface heat transfer increase with the acceleration, the magnetic and the rotation parameters whether the magnetic field is fixed relative to the fluid or body, except that the surface shear stress in $x$-direction and the surface heat transfer decrease with increasing the magnetic parameter when the magnetic field is fixed relative to the body. For a certain value of the acceleration parameter, the surface shear stress in the $x$-direction vanishes while the surface shear stress in the $y$-direction and the surface heat transfer remain finite. Also, below a certain value of the acceleration parameter, reverse flow occurs in the $x$-component of the velocity profile.
\end{abstract}

\section{1}

\section{Introduction}

The flow and heat transfer characteristics of spinning bodies of revolution in a forced flow stream are important in a variety of applications in engineering such as re-entry of missiles, projectile motion, fibre coating, and rotating machinery design. Reviews on this topic were written by Dorfiman [1] and Kraith [2]. The flow and heat transfer on a rotating sphere in a uniform flow stream with its axis of rotation parallel to the free stream velocity have been studied by a number of investigators [3-8]. The above

Received on 18 May 1998

H. S. Takhar

School of Engineering,

University of Manchester, Manchester,

M13 9PL. U.K.

G. Nath

Department of Mathematics,

Indian Institute of Science,

Bangalore-560012, India

Correspondence to: H. S. Takhar studies deal with the steady flows. The analogous unsteady problem was considered by Kumari and Nath [9] with the time-dependent free stream velocity. Ece [10] on the other hand studied the unsteady flow past an impulsively started translating and spinning sphere.

It may be remarked that most exact solutions in fluid mechanics and MHD are similarity solutions in the sense that the number of independent variables is reduced by one or more. These similarity solutions are derived i) by dimensional arguments, ii) by the group-theoretic method and iii) by the adhoc method of the free parameters.

Among these methods the group-theoretic method which includes dimensional analysis as a special case, is the most systematic in generating similarity solutions. These methods are extensively described in references [11-16].

Here we have studied the unsteady flow and heat transfer of a viscous electrically conducting fluid in the forward stagnation-point region of a rotating sphere in the presence of a magnetic field applied in the direction normal to the surface. Two cases have been considered, namely (a) when the magnetic field is fixed relative to the fluid and (b) when it is fixed relative to the body. The unsteadiness in the flow and the temperature fields is induced by the velocity at the edge of the boundary layer and by the angular velocity of the sphere, which vary continuously with time. It is found that a self-similar solution exists if the velocity at the edge of the boundary layer varies directly as the distance $x$ and inversely as the time $t$ and also the angular velocity of the sphere varies inversely as the time $t$. The system of ordinary differential equations governing the flow and temperature fields is solved numerically using a shooting method. Some analytical solutions are also obtained. Particular cases of the present results are compared with these of Lee et al. [7] and Sparrow et al. [17]. It may be remarked that in recent years the study of the interaction of electromagnetic fields with fluids has become important due to the possibility of applications in areas like nuclear fusion, chemical engineering, medicine and high speed noiseless printing.

2

\section{Problem formulation}

We consider the unsteady motion of a viscous, incompressible electrically conducting fluid in the forward stagnation-point region of a rotating sphere. The unsteadiness in the flow field is caused by the velocity at the edge of the boundary layer $\left(u_{e}=A x / t, A>0, x>0, x>0, t>0\right)$ and the angular 


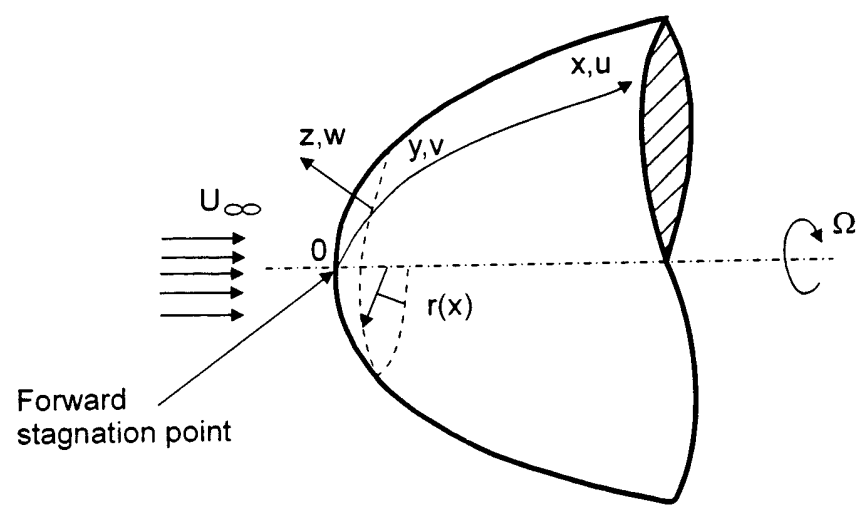

Fig. 1. Physical model and the co-ordinate system

velocity of the sphere $(\Omega=C / t, C>0, t>0)$, both varying inversely as time $t$. Fig. 1 shows the orthogonal curvilinear co-ordinate system in which $x$ measures the distance along a meridian from the forward stagnation point, $y$ represents the distance in the direction of rotation and $z$ is the distance normal to the surface, $u, v$ and $w$ are the components of velocity in $x$-, $y$-, and $z$-directions, respectively. It is assumed that the flow is axisymmetric. Hence the velocity components $(u, v, w)$, temperature $(T)$ and static pressure $(p)$ are independent of $y ; r(x)$ is the distance of a point on the body from the axis of rotation and it is equal to $x$ in the vicinity of the stagnation point. A uniform magnetic field of strength $B$ is applied to the boundary layer in the $z$-direction, which is fixed relative to either the fluid or the body. It is assumed that the magnetic Reynolds number $\mathrm{R}_{\mathrm{m}}=\mu_{0} \sigma V L \ll 1$, where $\mu_{0}$ is the magnetic permeability, $\sigma$ is the electrical conductivity, and $V$ and $L$ are respectively, the characteristic velocity and length. Under this condition, it is possible to neglect the effect of the induced magnetic field. It is also assumed that there is no applied polarization voltage which implies that the electric field $\mathbf{E}=0$. Hence the electrical term is not included in the relevant equations. This corresponds to the case where no energy is added to or extracted from the fluid by electrical means. The electrical current flowing in the fluid will give rise to an induced magnetic field which would exist if the fluid were an electrical insulator. Here we have taken the fluid as electrically conducting. When the magnetic field is fixed relative to the body only the fluid within the boundary layer is electrically conducting. The viscous dissipation terms, ohmic heating and surface curvature are neglected in the vicinity of the stagnation point. The wall and free stream temperatures are taken as constant. Under the above assumptions, the boundary layer equations governing the unsteady flow in the front stagnation point region of a rotating sphere in the presence of a magnetic field can be expressed as [7-10]

$$
\begin{aligned}
& \frac{\partial}{\partial x}(u x)+\frac{\partial}{\partial z}(w x)=0 \\
& \frac{\partial u}{\partial t}+u \frac{\partial u}{\partial x}+w \frac{\partial u}{\partial z}-\frac{v^{2}}{x} \\
& =u_{e} \frac{\partial u_{e}}{\partial x}+\frac{\partial u_{e}}{\partial t}+v \frac{\partial^{2} u}{\partial z^{2}}-\frac{\sigma \mathbf{B}^{2}}{\rho}\left(u-b u_{e}\right)
\end{aligned}
$$

$$
\begin{aligned}
& \frac{\partial v}{\partial t}+u \frac{\partial v}{\partial x}+w \frac{\partial v}{\partial z}+\frac{u v}{x}=v \frac{\partial^{2}}{\partial z^{2}}-\frac{\sigma \mathbf{B}^{2}}{\rho} v, \\
& \frac{\partial T}{\partial t}+u \frac{\partial T}{\partial x}+w \frac{\partial T}{\partial z}=\alpha \frac{\partial^{2} T}{\partial z^{2}}
\end{aligned}
$$

The initial conditions (i.e. at $t=0$ ) are given by;

$$
\begin{aligned}
u(x, z, 0) & =u_{i}(x, z), v(x, z, 0) \\
& =v_{i}(x, z), w(x, z, 0) \\
& =w_{i}(x, z), T(x, z, 0) \\
& =T_{i}(x, z) .
\end{aligned}
$$

and the boundary conditions are given by

$$
\begin{aligned}
u(x, 0, t) & =0, v(x, 0, t)=\Omega x, w(x, 0, t) \\
& =0, T(x, 0, t)=T_{w}, \\
u(x, \infty, t) & =u_{e}(x, t), v(x, \infty, t) \\
& =0, T(x, \infty, t)=T_{\infty} .
\end{aligned}
$$

Here $\rho$ and $v$ are, respectively fluid density and kinematics viscosity; $b$ is a dimensionless constant and $b=1$ or zero according to whether the magnetic field $\mathbf{B}$ is fixed relative to the fluid or body; $\alpha$ is the thermal diffusivity; $\Omega$ is the angular velocity of the rotating sphere; and the subscripts $e, i, w$ and $\infty$ denote condition at the edge of the boundary layer, initial condition, condition at the surface and free stream condition, respectively.

Equations (1)-(4) are a system of partial differential equations with three independent variables $x, z$ and $t$. It is shown that these partial differential equations can be reduced to a system of ordinary differential equations if we take the velocity at the edge of the boundary layer $u_{e}$ to be varying directly as the distance $x$ and inversely as time $t$ and the angular velocity of the sphere to the varying inversely as time $t$. Consequently, we apply the following transformations

$u_{e}=A x / t, A>0, x>0, t>0, \Omega=C / t, C>0$,

$\eta=2^{1 / 2}(v t)^{-1 / 2} z, u=(A x / t) f^{\prime}(\eta)$,

$v=(C x / t) s(\eta), w=-2^{1 / 2}(v / t)^{1 / 2} A f(\eta)$,

$T-T_{w}=\left(T_{\infty}-T_{w}\right) g(\eta), M=H^{2} a / \operatorname{Re}_{x}$,

$H^{2} a=\sigma \mathbf{B}^{2} x^{2} / \mu$,

$\operatorname{Re}_{x}=u_{e} x / v, \lambda=(C / A)^{2}=\left(v_{w} / u_{e}\right)^{2}, \operatorname{Pr}=v / \alpha$,

to Eqs (1)-(4) and we find that Eq. (1) is identically satisfied and Eqs (2)-(4) reduce to

$$
\begin{aligned}
& f^{\prime \prime \prime}+A f f^{\prime \prime}+2^{-1} A\left[1-\left(f^{\prime}\right)^{2}+\lambda s^{2}\right]-2^{-1} \\
& \quad \times\left(1-f^{\prime}-\eta f^{\prime \prime} / 2\right)-2^{-1} A M\left(f^{\prime}-b\right)=0, \\
& s^{\prime \prime}+A\left(f s^{\prime}-f^{\prime} s\right)+2^{-1}\left(s+\eta s^{\prime} / 2\right)-2^{-1} A M s=0, \\
& \operatorname{Pr}^{-1} g^{\prime \prime}+A f g^{\prime}+(\eta / 4) g^{\prime}=0 .
\end{aligned}
$$

The boundary conditions (6) can be rewritten in the form $f(0)=f^{\prime}(0)=0, s(0)=1, g(0)=0$,

$f^{\prime}(\infty)=1, s(\infty)=0, g(\infty)=1$. 
Here $\eta$ is the dimensionless similarity variable; $f^{\prime}$ and $s$ are the dimensionless velocity components along $x$ - and $y$-directions, respectively; $f$ is the component of velocity in $z$-direction; $g$ is the dimensionless temperature; $\mathrm{Pr}$ is the Prandtl number; $M$ is the magnetic parameter; $\mathrm{Ha}$ is the Hartmann number; $\mathrm{Re}_{x}$ is the local Reynolds number; $\mu$ is the coefficient of dynamic viscosity; $A$ and $C$ are the dimensionless positive constants and are associated, respectively, with the velocity at the edge of the boundary layer $\left(u_{e}=A x / t\right)$ and the angular velocity of the sphere $(\Omega=C / t)$ and prime denotes derivative with respect to $\eta$. $\lambda$ is the dimensionless rotation parameter and $\lambda=0$ implies that the sphere is stationary. Since there is no rotational motion of the sphere to induce a circumferential velocity in the fluid through the action of viscosity, the velocity component $v$ is identically zero. Hence, the momentum equation in $y$-direction (9) vanishes and the momentum equation in $x$-direction (8) reduces to that of the momentum equation in $x$-direction over a stationary sphere.

It may be remarked that the self-similar solution exists for a slightly different distribution of the velocity at edge of the boundary layer (i.e. $\left.u_{e}=a x\left(1-\alpha^{*} t^{*}\right)^{-1}\right)$ and of the angular velocity of the sphere (i.e. $\left.\Omega=\Omega_{0}\left(1-\alpha^{*} t^{*}\right)^{-1}\right)$. In this case, we apply the following transformations

$$
\begin{aligned}
\xi= & 2^{1 / 2}(a / v)^{1 / 2}\left(1-\alpha^{*} t^{*}\right)^{-1 / 2} z, \alpha^{*} t^{*}<1, t^{*}=a t, \\
\Omega= & \Omega_{0}\left(1-\alpha^{*} t^{*}\right)^{-1}, \\
u_{e}= & a x\left(1-\alpha^{*} t^{*}\right)^{-1}, a=\left(\partial u_{\mathrm{e}} / \partial x\right)_{t^{*}=0}, \\
u= & a x\left(1-\alpha^{*} t^{*}\right)^{-1} F^{\prime}(\xi), \\
v= & \Omega_{0} x\left(1-\alpha^{*} t^{*}\right)^{-1} S(\xi), \\
w= & -2^{1 / 2}(a v)^{1 / 2}\left(1-\alpha^{*} t^{*}\right)^{-1 / 2} F(\xi), \\
\lambda= & \left(\Omega_{0} / a\right)^{2}, \mathbf{B}=\mathbf{B}_{0} \\
& \quad \times\left(1-\alpha^{*} t^{*}\right)^{-1 / 2}, M=\sigma \mathbf{B}_{o}^{2} /(\rho a), \\
T- & T_{w}=\left(T_{\infty}-T_{w}\right) G(\xi),
\end{aligned}
$$

to Eqs. (1)-(4) and we find that Eq. (1) is satisfied identically and Eqs (2)-(4) reduce to the following system of equations

$$
\begin{aligned}
& F^{\prime \prime \prime}+F F^{\prime \prime}+2^{-1}\left[1-\left(F^{\prime}\right)^{2}+\lambda S^{2}\right] \\
& +2^{-1} \alpha^{*}\left(1-F^{\prime}-\xi F^{\prime \prime} / 2\right)+2^{-1} M\left(b-f^{\prime}\right)=0, \\
& S^{\prime \prime}+\left(F S^{\prime}-F^{\prime} S\right)-2^{-1} \alpha^{*}\left(S+\xi S^{\prime} / 2\right)-2^{-1} M S=0, \\
& \operatorname{Pr}^{-1} F^{\prime \prime}+F G^{\prime}-\alpha^{*}(\xi / 4) G^{\prime}=0 \text {. }
\end{aligned}
$$

The boundary conditions (6) are given by

$$
\begin{aligned}
F(0)=F^{\prime}(0) & =G(0)=0, S(0)=1, F^{\prime}(\infty)=G(\infty)=1, \\
S(\infty) & =0 .
\end{aligned}
$$

Here $\xi$ is the dimensionless similarly variable; $F^{\prime}$ and $S$ are the dimensionless velocity components in $x$ - and $y$-directions, respectively; $t^{*}$ is the dimensionless time; $G$ is the dimensionless temperature; $a$ is the velocity gradient at time $t^{*}=0 ; \alpha^{*}$ is the dimensionless parameter characteris- ing the unsteadiness in the flow field and $\alpha^{*}>0$, or $<0$ according to whether the flow is accelerating or decelerating and the prime here denotes derivatives with respect to $\xi$.

Equations (13)-(15) are slightly different from (8)-(10). However Eqs. (8)-(10) can be reduced to Eqs (13)-(15) if we apply the following transformations

$$
\begin{aligned}
\eta & =(A)^{-1 / 2} \xi, f(\eta) \\
& =(A)^{-1 / 2} F(\xi), s(\eta) \\
& =S(\xi) \\
g & (\eta)=G(\xi), A=-\left(\alpha^{*}\right)^{-1}, \alpha^{*}<0 .
\end{aligned}
$$

It may be noted that Eqs. (8)-(10) govern the unsteady flow in the forward stagnation-point region of a rotating sphere. The corresponding steady-state equations are obtained by omitting the terms representing the contribution to the unsteady flow. The steady-state equations are given by

$$
\begin{aligned}
& f^{\prime \prime \prime}+A f f^{\prime \prime}+2^{-1} A\left[1-\left(f^{\prime}\right)^{2}+\lambda s^{2}\right] \\
& \quad-2^{-1} A M\left(f^{\prime}-b\right)=0 \\
& s^{\prime \prime}+A\left(f^{\prime}-f^{\prime} s\right)-2^{-1} A M s=0 \\
& g^{\prime \prime}+\operatorname{Pr} A f g^{\prime}=0
\end{aligned}
$$

For $A=1, M=0$ Eqs (18)-(20) reduce to those of Lee et al. [7]. Also for $A=b=1, \lambda=0$, equations (18) and (20) reduce to those of Sparrow et al. [17] if we apply the following transformations

$\eta=2^{1 / 2} \xi, f(\eta)=2^{1 / 2} f_{1}(\xi), g(\eta)=g_{1}(\xi)$.

When $\lambda=0$ (no rotation), Eq. (19) is not required.

\section{3}

\section{Approximate solution}

It is possible to obtain an analytical (approximate) solution of equations (18) and (20) under conditions (11) for $\lambda=0, b=1$. We introduce the parameter $\beta$ for stretching both the independent variable $\eta$ and the dependent variable $f$. The new variables are given by

$\xi=\beta \eta, F(\xi)=\beta f(\eta), \beta>0$,

which transform the boundary value problem represented by equation (18) under conditions (11) into

$$
\begin{aligned}
\beta^{2} \frac{\mathrm{d}^{3} F}{\mathrm{~d} \xi^{3}} & +A F \frac{\mathrm{d}^{2} F}{\mathrm{~d} \xi^{2}}+2^{-1} A\left[1-\left(\frac{\mathrm{d} F}{\mathrm{~d} \xi}\right)^{2}\right] \\
& +2^{-1} A M\left(1-\frac{\mathrm{d} F}{\mathrm{~d} \xi}\right)=0,
\end{aligned}
$$

$F=\frac{\mathrm{d} F}{\mathrm{~d} \xi}=0$ at $\xi=0, \frac{\mathrm{d} F}{\mathrm{~d} \xi} \rightarrow 1$ as $\xi \rightarrow \infty$.

Now we take the approximation

$\mathrm{d} F / \mathrm{d} \xi=1-\exp (-\xi)$

which satisfies both the boundary conditions on $\mathrm{d} F / \mathrm{d} \xi$ in (24). Integration of (25) from zero to $\xi$ and using the boundary conditions on $F$ in (24) gives 
$F=\xi-1+\exp (-\xi)$.

The defect $R(\xi, \beta, A, M)$ in (23) is given by

$$
\begin{aligned}
R(\xi, \beta, A, M)= & \left(A \xi+2^{-1} A M-\beta^{2}\right) \exp (-\xi) \\
& +2^{-1} A \exp (-2 \xi) .
\end{aligned}
$$

We now apply the least square method to minimize $R$ in (27) and we get

$\frac{\partial}{\partial \beta} \int_{0}^{\infty} R^{2}(\xi, \beta, A, M) \mathrm{d} \xi=0$.

From the above equation, we get

$\beta=f^{\prime \prime}(0)=[(5 / 6) A+(A / 2) M]^{1 / 2}$.

The expressions for $f, f^{\prime}$ and $R$ in terms of $\eta$ are given by

$f=\eta-\beta^{-1}[1-\exp (-\beta \eta)]$

$f^{\prime}=1-\exp (-\beta \eta)$

$$
\begin{aligned}
R(\eta, \beta, A)= & {[A \beta \eta-(5 / 6) A \exp (-\beta \eta)} \\
& \left.+2^{-1} A \exp (-2 \beta \eta)\right] .
\end{aligned}
$$

Using the relation (30a) in (20) we get

$g^{\prime \prime}+\operatorname{Pr} A\left[\eta-\beta^{-1}(1-\exp (-\beta \eta))\right] g^{\prime}=0$.

The solution of (31) under boundary conditions (11) is given by

$g=\int_{0}^{\eta} P(\eta) \mathrm{d} \eta / \int_{0}^{\infty} P(\eta) \mathrm{d} \eta$

$g^{\prime}(0)=\left[\int_{0}^{\infty} P(\eta) \mathrm{d} \eta\right]^{-1}$

where

$$
\begin{aligned}
& P(\eta)=\exp [-A \operatorname{Pr}\left(2^{-1} \eta^{2}-\beta^{-1} \eta\right. \\
&\left.\left.+\beta^{-2}-\beta^{2} \exp (-\beta \eta)\right)\right]
\end{aligned}
$$

and $\beta$ in $P(\eta)$ is given by (29).

The results $\left(f^{\prime \prime}(0), g^{\prime}(0)\right)$ of the analytical solution are compared with the corresponding results of the numerical solution. For $M=1, A=1$ the difference is about 0.8 per cent for the surface shear stress $\left(f^{\prime \prime}(0)\right)$ and about 1.5 per cent for the surface heat transfer $\left(g^{\prime}(0)\right)$. This difference decreases as $M$ or $A$ increases.

\section{4}

\section{Asymptotic solution}

Here we consider the asymptotic behaviour of the solutions of equations (8)-(10) under conditions (11) for large $\eta$. As $\eta \rightarrow \infty, f^{\prime}$ and $g$ tend to 1 and $s \rightarrow 0$. Also

$(\eta-f) \rightarrow \int_{0}^{\infty}(1-f) \mathrm{d} \eta$
Since the above integral is the displacement thickness of the boundary layer, it must be finite and is usually small. This implies that $f \rightarrow \eta$ for large $\eta$. We set for large $\eta$.

$f(\eta)=\eta+f_{1}(\eta), s(\eta)=s_{1}(\eta), g(\eta)=1+g_{1}(\eta)$

where $f_{1}, s_{1}$ and $g_{1}$ are small and their squares and products can be neglected. Using (35) in (8)-(10), we get

$f_{1}^{\prime \prime \prime}+c_{1} \eta f_{1}^{\prime \prime}-c_{2}\left(f_{1}^{\prime}+\lambda\right)=0$

$s_{1}^{\prime \prime}+c_{1} \eta s_{1}^{\prime}-c_{2} s_{1}=0$

$g_{1}^{\prime \prime}+c_{1} \operatorname{Pr} \eta g_{1}^{\prime}=0$

where

$$
\begin{aligned}
c_{1}= & A+4^{-1}, c_{2}=A+2^{-1}(A M-1), A>0, c_{1}>0, \\
c_{3}= & 2^{-1} A M(1-b), \lambda=c_{3} / c_{2}, c_{2}>0 \text { for } \\
& A>(2+M)^{-1} .
\end{aligned}
$$

The boundary conditions on $f_{1}^{\prime}, s_{1}$ and $g_{1}$ for $\eta \rightarrow \infty$ are given by

$f_{1}^{\prime}, s_{1}, g_{1} \rightarrow 0$ as $\eta \rightarrow \infty$.

Equations (36a) and (36b) admit solutions in terms of parabolic cylinder functions [18].

$$
\begin{aligned}
f_{1}^{\prime}+\lambda=s_{1}= & \exp \left(-c_{1} \eta^{2} / 4\right)\left[B_{1} D_{1}\left(c_{1}^{1 / 2} \eta\right)\right. \\
& \left.+B_{2} D_{2}\left(i c_{1}^{1 / 2} \eta\right)\right]
\end{aligned}
$$

where

$D_{1}\left(c_{1}^{1 / 2} \eta\right)=\exp \left(-c_{1} \eta^{2} / 4\right)\left(c_{1}^{1 / 2} \eta\right)^{-c_{4}-1}\left[1+0\left(c_{1}^{-1} \eta^{-2}\right)\right]$

$D_{2}\left(i c_{1}^{1 / 2} \eta\right)=\exp \left(c_{1} \eta^{2} / 4\right)\left(c_{1}^{1 / 2} \eta\right) c_{4}\left[1+0\left(c_{1}^{-1} \eta^{-2}\right)\right]$

$c_{4}=c_{2} / c_{1}, c_{1}>0$

and $B_{1}$ and $B_{2}$ are arbitrary constants. In view of the conditions $(36 \mathrm{e})$, the divergent term $D_{2}\left(i c_{1}^{1 / 2} \eta\right)$ must be omitted for $c_{4}>0$ (i.e. $c_{2}>0$ ). Hence the constant $B_{2}=0$ and from (37a) the solution for $f_{1}^{\prime}$ and $s_{1}$ can be expressed as

$$
\begin{aligned}
f_{1}^{\prime}+\lambda=s_{1}= & B_{1} \exp \left(-c_{1} \eta^{2} / 2\right)\left(c_{1}^{1 / 2} \eta\right)^{-c_{4}-1} \\
& \times\left[1+0\left(c_{1}^{-1} \eta^{-2}\right)\right] .
\end{aligned}
$$

The solution of (36c) under the boundary, condition on $g_{1}$ in (36e), is given by

$$
\begin{aligned}
g_{1}= & -B_{3}\left(\operatorname{Pr} c_{1} \eta\right)^{-1} \exp \left(-\operatorname{Pr} c_{1} \eta^{2} / 2\right) \\
& \left.\times\left[1-\operatorname{Pr} c_{1} \eta^{2}\right)^{-1}+\ldots\right]
\end{aligned}
$$

where $B_{3}$ is an arbitrary constant. For $c_{4}<0$ (i.e. $c_{2}>0$ ) the solutions for $f_{1}^{\prime}$ and $s_{1}$ are given by (37a) in the form

$$
\begin{aligned}
f_{1}^{\prime}+\lambda=s_{1}= & B_{1} \exp \left(-c_{1} \eta^{2} / 2\right)\left(c_{1}^{1 / 2} \eta\right)^{-c_{4}-1} \\
& \times\left[1+0\left(c_{1}^{-1} \eta^{-2}\right)\right] \\
& +B_{2}\left(c_{1}^{1 / 2} \eta\right)^{c_{4}}\left[1+0\left(c_{1}^{-1} \eta^{-2}\right)\right] .
\end{aligned}
$$

It is evident from equations (38a) and (38c) along with (35) that the velocity profiles in $x$-direction $\left(f^{\prime}\right)$ tends to 1 in an exponential manner for large $\eta$ only when $b=1$ $(\lambda=0)$. For $b=0(\lambda>0) f^{\prime}\left(f^{\prime}=1+f^{\prime}\right)$ does not tend to 1 
exponentially. However, the velocity profile in $y$-direction $s$ and the temperature profile $g$ tend to their edge values (i.e., 0 and 1, respectively) exponentially for both $b=0$ and 1 . The physical reason for this is that for $b=0$, the viscous fluid in the boundary layer is electrically conducting, but the fluid outside the boundary layer is nonconducting. Therefore, there is no magnetic field outside the boundary layer. This discontinuity of the magnetic field for $b=0$ is responsible for the non-exponential behaviour of the velocity profile $f^{\prime}$ for large $\eta$. For $c_{2}=0$ (i.e. $c_{4}=0$ ), Eqs. (36a) and (36b) are similar to (36c). Hence for $c_{2}=0$ the solutions for $f_{1}^{\prime}$ and $s_{1}$ are given by (38b) with $\operatorname{Pr}=1$.

\section{5}

\section{Results and discussion}

Equations (8)-(10) under the boundary conditions (11) are solved numerically using a double shooting method which is described in detail in [19]. The results are obtained for various values of the parameters $M(0 \leq M \leq 4), \lambda(0 \leq \lambda \leq 5), A(0.1 \leq A \leq 2)$. However for the sake of brevity, only some representative results are presented here.

In order to assess the accuracy of our method, we have compared our results for the surface shear stresses in $x$-and $y$-directions $\left(f^{\prime \prime}(0),-s^{\prime}(0)\right)$ and the surface heat transfer $\left(g^{\prime}(0)\right.$ corresponding to the steady-state case when $A=1, M=0$ with the tabulated results of Lee et al. [7]. Also, for $A=b=1, \lambda=0$ the results for the surface heat transfer $\left(g^{\prime}(0)\right)$ for the steady-state case have been compared with the tabulated results of Sparrow et al. [17]. For direct comparison we have to multiply our results for $g^{\prime}(0)$ by $2^{1 / 2}$. In both cases the results are found to agree within 0.2 per cent. Hence for the sake of brevity the comparison is not shown here. The results for $b=1$ (i.e., when the magnetic fixed $\mathbf{B}$ is fixed relative to the fluid) are given in Figs. 2-5 and Tables 1 and 2 and for $b=0$ (i.e., when the magnetic field $\mathbf{B}$ is fixed relative to the body) in Tables 3 and 4 and in Fig. 6.

The effect of the acceleration parameter $A$ on the velocity profiles in $x$ - and $y$-direction $\left(f^{\prime}, s\right)$ and the temperature profiles $(g)$ for $\lambda=M=1, \operatorname{Pr}=0.7$ is shown in

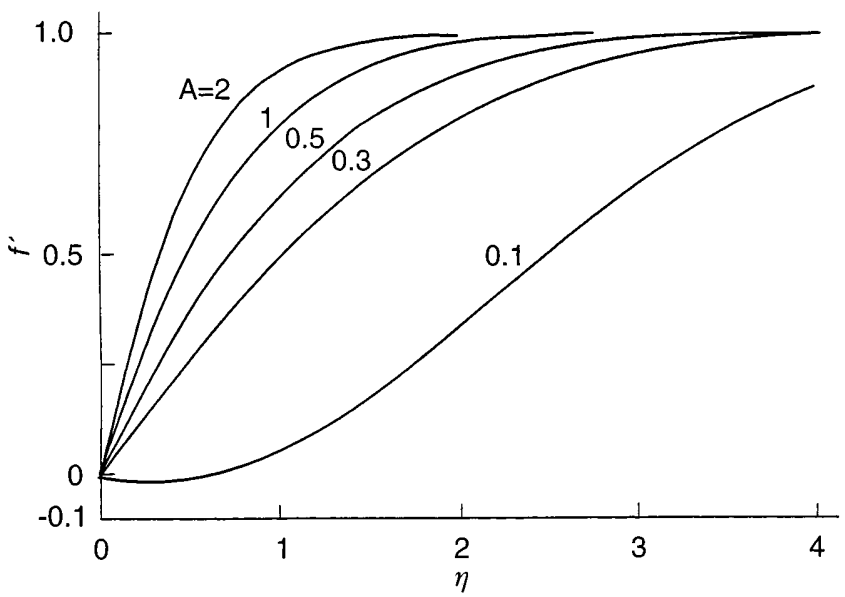

Fig. 2. Velocity profiles in $x$-direction $\left(f^{\prime}\right)$ for $A=0.1,0.3,0.5$, $1,2, M=2, \lambda=b=1$
Figs. 2 and 3. It is observed from these figures that the velocity profiles in the $x$-direction $\left(f^{\prime}\right)$ and the temperature profiles $(g)$ increase everywhere with increasing $A$, but the viscous and thermal boundary layerthickness decrease.

The physical reason for this behaviour is that the velocity

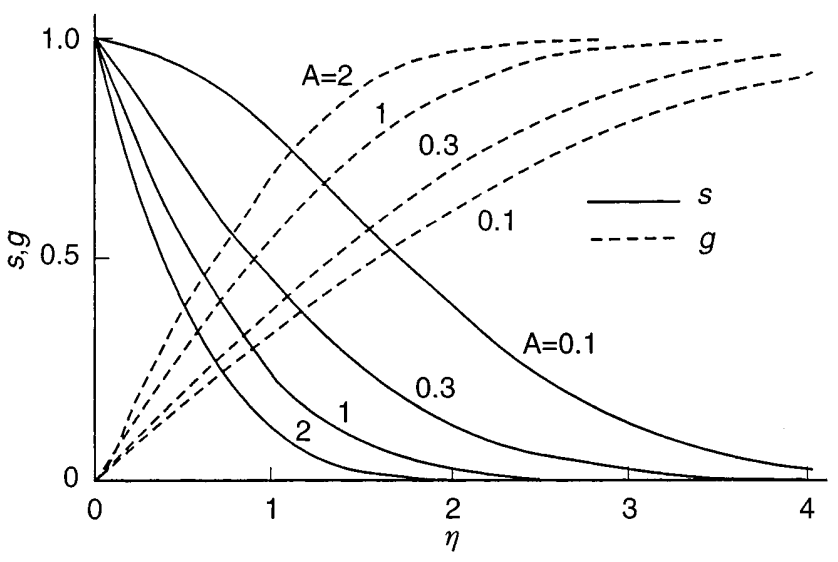

Fig. 3. Velocity profiles in $y$-direction (s) and temperature profiles $(g)$ for $A=0.1,0.3,1,2, M=2, \lambda=b=1, \operatorname{Pr}=0.7$

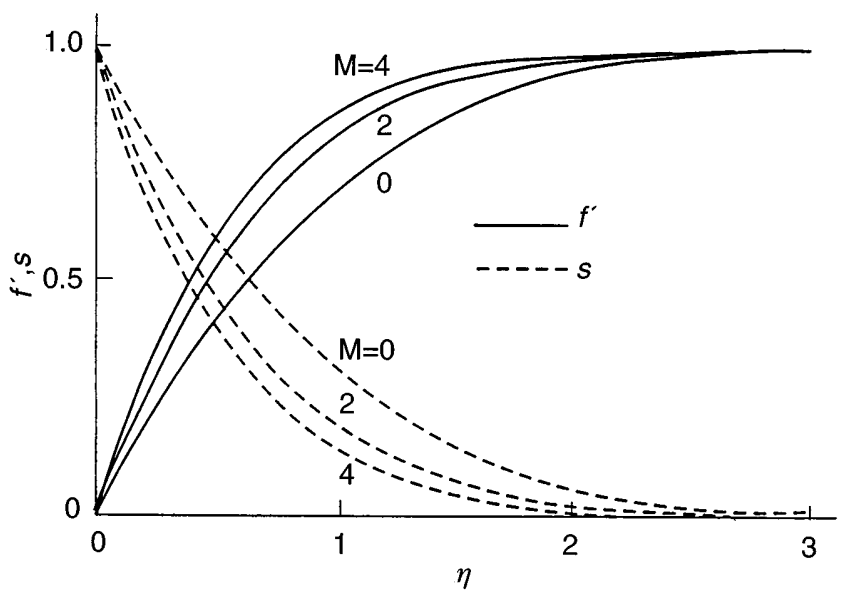

Fig. 4. Velocity profiles in $x$ - and $y$-directions $\left(f^{\prime}, s\right)$ for $M=0$ $2,4, A=\lambda=b=1$

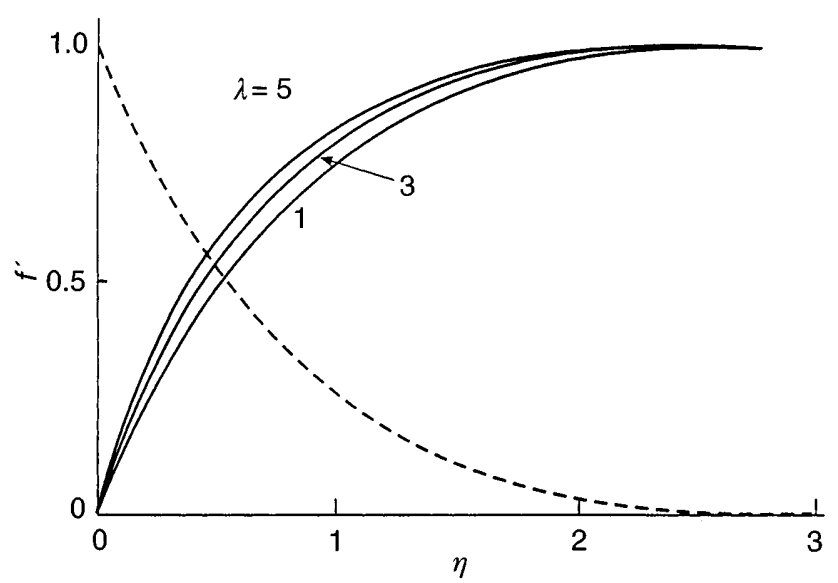

Fig. 5. Velocity profile in $x$-directions $\left(f^{\prime}\right)$ for $\lambda=1,3,5$, $A=M=b=1$ 
Table 1. Surface shear stresses in $x$ - and $y$-directions $\left(f^{\prime \prime}(0),-s^{\prime}\right.$ $(0))$ and the surface heat transfer $\left(g^{\prime}(0)\right)$ for $\lambda=1, \operatorname{Pr}=0.7, b=1$

\begin{tabular}{lllll}
\hline$M$ & $A$ & $f^{\prime \prime}(0)$ & $-s^{\prime}(0)$ & $g^{\prime}(0)$ \\
\hline 0 & 0.3 & 0.1818 & 0.7493 & 0.3886 \\
0 & 0.5 & 0.4880 & 0.8271 & 0.4450 \\
0 & 1.0 & 0.9233 & 0.9381 & 0.5611 \\
0 & 2.0 & 1.4655 & 1.0989 & 0.7418 \\
1 & 0.3 & 0.3924 & 0.8738 & 0.4018 \\
1 & 0.5 & 0.6897 & 0.9805 & 0.4582 \\
1 & 1.0 & 1.1552 & 1.1733 & 0.5766 \\
1 & 2.0 & 1.7578 & 1.4664 & 0.7612 \\
2 & 0.1 & -0.1047 & 0.0282 & 0.3442 \\
2 & 0.3 & 0.5566 & 0.5639 & 0.4103 \\
2 & 0.5 & 0.8624 & 0.7674 & 0.4677 \\
2 & 1.0 & 1.3624 & 1.1065 & 0.5886 \\
2 & 2.0 & 2.0240 & 1.5695 & 0.7768 \\
3 & 0.1 & 0.0391 & 0.2059 & 0.3492 \\
3 & 0.3 & 0.6720 & 0.6823 & 0.4155 \\
3 & 0.5 & 0.9933 & 0.9107 & 0.4741 \\
3 & 1.0 & 1.5209 & 1.3051 & 0.5972 \\
3 & 2.0 & 2.2514 & 1.8507 & 0.7886 \\
4 & 0.1 & 0.1444 & 0.3151 & 0.3525 \\
4 & 0.3 & 0.7723 & 0.7837 & 0.4197 \\
4 & 0.5 & 1.1099 & 1.0358 & 0.4791 \\
4 & 1.0 & 1.6838 & 1.4797 & 0.6042 \\
4 & 2.0 & 2.4597 & 2.0977 & 0.7984 \\
\hline
\end{tabular}

Table 2. Surface shear stresses in $x$ - and $y$-directions $\left(f^{\prime \prime}(0),-s^{\prime}\right.$ $(0))$ and the surface heat transfer $\left(g^{\prime}(0)\right)$ for $M=A=2, \operatorname{Pr}=0.7$, $b=1$

\begin{tabular}{llll}
\hline$\lambda$ & $\mathrm{f}^{\prime \prime}(0)$ & $-s^{\prime}(0)$ & $g^{\prime}(0)$ \\
\hline 0 & 1.0062 & - & 0.5697 \\
1 & 1.1552 & 1.1733 & 0.5766 \\
2 & 1.3008 & 1.1846 & 0.5832 \\
3 & 1.4434 & 1.1953 & 0.5832 \\
4 & 1.5832 & 1.2056 & 0.5954 \\
5 & 1.7205 & 1.2154 & 0.6011 \\
\hline
\end{tabular}

Table 3. Surface shear stresses in $x$ - and $y$-directions $\left(f^{\prime \prime}(0),-s^{\prime}\right.$ $(0))$ and the surface heat transfer $\left(g^{\prime}(0)\right)$ for $\lambda=1, \operatorname{Pr}=0.7$, $b=0$

\begin{tabular}{llrll}
\hline$M$ & $A$ & \multicolumn{1}{c}{$f^{\prime \prime}(0)$} & \multicolumn{1}{c}{$-s^{\prime}(0)$} & $g^{\prime}(0)$ \\
\hline 1 & 0.5 & -0.0746 & 0.7919 & 0.3497 \\
1 & 0.75 & 0.2205 & 0.9302 & 0.4095 \\
1 & 1.0 & 0.4210 & 1.0383 & 0.4575 \\
1 & 1.5 & 0.6901 & 1.2145 & 0.5611 \\
1 & 2.0 & 0.8950 & 1.3365 & 0.6142 \\
3 & 0.75 & -0.0138 & 0.8432 & 0.3014 \\
3 & 1.0 & 0.1570 & 1.0820 & 0.3543 \\
3 & 1.5 & 0.3679 & 1.4138 & 0.4247 \\
3 & 2.0 & 0.5149 & 1.6730 & 0.4808 \\
4 & 0.75 & -0.0159 & 1.0478 & 0.3054 \\
4 & 1.0 & 0.1291 & 1.2865 & 0.3448 \\
4 & 1.5 & 0.3112 & 1.6473 & 0.4036 \\
4 & 2.0 & 0.4400 & 1.9370 & 0.4520 \\
\hline
\end{tabular}

at the edge of the boundary layer $u_{e}$ increases as $A$ increases. This imports an additional momentum in the $x$ direction into the boundary layer and the fluid inside the boundary layer gets accelerated in $x$-direction which in-
Table 4. Surface shear stresses in $x$ - and $y$-directions $\left(f^{\prime \prime}(0)\right.$, $\left.-s^{\prime}(0)\right)$ and the surface heat transfer $\left(g^{\prime}(0)\right)$ for $M=A=1$, $\operatorname{Pr}=0.7, b=0$

\begin{tabular}{llll}
\hline$\lambda$ & $f^{\prime \prime}(0)$ & $-s^{\prime}(0)$ & $g^{\prime}(0)$ \\
\hline 0 & 0.2315 & - & 0.4416 \\
1 & 0.4210 & 1.0383 & 0.4575 \\
2 & 0.5993 & 1.0583 & 0.4712 \\
3 & 0.7691 & 1.0762 & 0.4832 \\
4 & 0.9322 & 1.0925 & 0.4941 \\
5 & 1.0897 & 1.1075 & 0.5039 \\
\hline
\end{tabular}

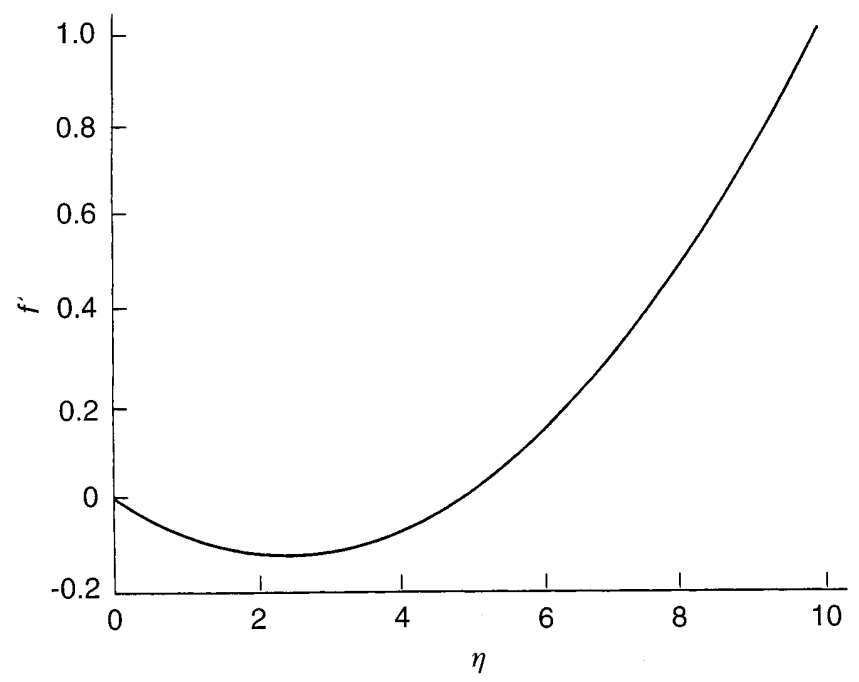

Fig. 6. Velocity profiles in $x$-directions $\left(f^{\prime}\right)$ for $A=0.75, M=3$, $\lambda=1, b=0$

creases the velocity $f^{\prime}$ and hence the temperature $g$, and reduces the viscous and thermal boundary layer thicknesses. On the other hand, increasing A tends to oppose the fluid motion in the rotating direction (i.e $y$-direction) as is evident from equation (9). Consequently, the velocity in the rotating direction is everywhere lowered and the boundary layer in the rotating direction becomes thin. For $A=0.1$, the reverse flow is observed in $f^{\prime}$ near the wall. The reason for this behaviour is given later.

The effect of the magnetic parameter $M$ on the velocity profiles in $x$ - and $y$-directions $\left(f^{\prime}, \mathrm{s}\right)$ for $\lambda=A=1$ is presented in Fig. 4. Since the effect of $M$ on the temperature profiles $(g)$ is very small, it is not shown here. The magnetic field $\mathbf{B}$ induces a magnetic force in the $x$-direction which tends to support the velocity in that direction. Hence the $x$-component of the velocity profiles $\left(f^{\prime}\right)$ is everywhere increased. Consequently, the temperature profile $(g)$ is also increased. The viscous boundary layer thickness in the $x$-direction and the thermal boundary layer thickness are reduced with an increasing $M$. On the other hand, the magnetic field gives rise to a magnetic force in the rotating direction (i.e $y$-direction) which tends to oppose the velocity in that direction. Hence the velocity in $y$-direction is reduced and the boundary layer thickness is thinned as illustrated in Fig. $3 \mathrm{~b}$.

The effect of the rotation parameter $\lambda$ on the velocity profiles in the $x$-direction $\left(f^{\prime}\right)$ is shown in Fig. 5 . Since the 
effect of $\lambda$ on the velocity profiles in the $y$-direction (s) and the temperature profiles $(g)$ is found to be small because $\lambda$ affects them indirectly, these profiles are not shown here. The increase in $\lambda$ injects an additional momentum into the boundary layer which accelerates the fluid. Hence the velocity profiles in $x$-direction are increased and the boundary layer thickness decreases.

The effect of the acceleration parameter $A$ on the surface shear stresses in $x$ - and $y$-directions $\left(f^{\prime \prime}(0),-s^{\prime}(0)\right)$ and the surface heat transfer $\left(g^{\prime}(0)\right)$ for $\operatorname{Pr}=0.7$ is presented in Table 1. As mentioned earlier, both the viscous and thermal boundary layer thicknesses increase with decreasing $\lambda$. Consequently, the surface shear stresses and the surface heat transfer $\left(f^{\prime \prime}(0),-s^{\prime}(0), g^{\prime}(0)\right)$ decrease with decreasing $A$. For example, for $\lambda=M=1, \operatorname{Pr}=0.7$, $f^{\prime \prime}(0),-s^{\prime}(0)$ and $g^{\prime}(0)$ decrease by about $77 \%, 40 \%$ and $47 \%$, respectively, when $A$ decreases from 2 to 0.3 . The interesting result is that the surface shear stress in $x$-direction $f^{\prime \prime}(0)$ vanishes for a certain value of $A=A_{0}$ which depends on $M$ and $\lambda$, but the surface shear stress in $y$ direction and the surface heat transfer $\left(-s^{\prime}(0), g^{\prime}(0)\right)$ remain finite. $A_{0}=0.2061,0.1466,0.1265,0.0932,0.0671$ for $M=0,1,2,3,4$ respectively, when $\lambda=1$. Similar results have been obtained by Yang [20] in the case of the unsteady flow in the stagnation-point region of a twodimensional body without a magnetic field. Unlike the steady case, the vanishing of the surface shear stress $\left(f^{\prime \prime}(0)\right)$ does not imply separation $[21,22]$. Hence, when $A$ is slightly further reduced $f^{\prime \prime}(0)<0$ which implies that there is a reverse flow in the $x$-component of the velocity profile $\left(f^{\prime}\right)$ as shown in Fig. 2.

The effect of the magnetic parameter $M$ and the rotation parameter $\lambda$ on the surface shear stresses and the surface heat transfer $\left(f^{\prime \prime}(0),-s^{\prime}(0), g^{\prime}(0)\right)$ for $\operatorname{Pr}=0.7$ is shown in Tables 1 and 2. The surface shear stresses and the surface heat transfer $\left(f^{\prime \prime}(0),-s^{\prime}(0), g^{\prime}(0)\right)$ increase with $M$ or $\lambda$ due to the reduction of viscous and thermal boundary layers as mentioned earlier. However, the effect of $M$ on $g^{\prime}(0)$ and the effect of $\lambda$ on $-s^{\prime}(0)$ and $g^{\prime}(0)$ are small. For $\lambda=A=1, \operatorname{Pr}=0.7, f^{\prime \prime}(0),-s^{\prime}(0)$ and $g^{\prime}(0)$ increase, respectively, by about $82 \%, 80 \%$ and $7.6 \%$ when $M$ increases from zero to 4. Also for $A=M=1, \operatorname{Pr}=0.7$, $f^{\prime \prime}(0),-s^{\prime}(0), g^{\prime}(0)$ increase by about $50 \%, 3.6 \%, 4.2$, respectively, as $\lambda$ increases from 1 to 5 .

We have also obtained the results for the case when the magnetic field $\mathbf{B}$ is fixed relative to the body $(b=0)$. Since the effects of $A$ and $\lambda$ on the velocity and temperature profiles $\left(f^{\prime}, s, g\right)$ and the effect of $M$ on the velocity profiles (s) are found to be qualitatively similar to those of the previous case $(b=1)$, for the sake of brevity these are not presented here. The effect of the magnetic parameter $M$ on the velocity profiles in the $x$-direction $\left(f^{\prime}\right)$ and the temperature profiles $(g)$ is found to be opposite to that of the previous case $(b=1)$. For $b=0$ the magnetic field induces a magnetic force in the $x$-direction which tends to oppose the velocity $f^{\prime}$. This reduces the velocity $f^{\prime}$ and hence the temperature $g$. The viscous and thermal boundary layers increase with increasing $M$. Also, for $b=0$ and $M>0$ the velocity profiles in $x$-direction $\left(f^{\prime}\right)$ do not tend to 1 in an exponential manner when $\eta \rightarrow \infty$ as mentioned in the previous section.
The effect of the acceleration parameter $A$ on the surface shear stresses in the $x$ - and $y$-directions $\left(f^{\prime \prime}(0),-s^{\prime}(0)\right)$ and the surface heat transfer $\left(g^{\prime}(0)\right)$ is presented in Table 3. Qualitatively, they are similar to the previous case $(b=1)$. However, $f^{\prime \prime}(0)$ is much less than that of the previous case. For $M=\lambda=1, f^{\prime \prime}(0)$ is about $1 / 3$ of the corresponding value of case $\mathrm{I}(b=1)$. The value of $A=A_{0}$ for which $f^{\prime \prime}(0)=0$ is more than that of the case I is given by $A_{0}=0.5466,0.7204,0.7667,0.7939$ for $M=1,2,3,4$, respectively. Below these values of $A_{0}, f^{\prime \prime}(0)<0$, which implies that reverse flow occurs in the velocity profile $f^{\prime}$ as shown in Fig. 6 . As mentioned earlier for $M>0, b=0, f^{\prime}$ does not tend to 1 in an exponential manner at the edge of the boundary layer.

The effect of the magnetic parameter $M$ and the rotation parameter $\lambda$ on the surface shear stresses and heat transfer $\left(f^{\prime \prime}(0),-s^{\prime}(0), g^{\prime}(0)\right)$ for $\operatorname{Pr}=0.7$ is displayed on Tables 3 and 4 . The surface shear stress in the $x$-direction $\left(f^{\prime \prime}(0)\right)$ and the surface heat transfer $\left(g^{\prime}(0)\right)$ decrease with increasing $M$ due to the thickening of viscous and thermal boundary layers. For $A=\lambda=1, \operatorname{Pr}=0.7, f^{\prime \prime}(0)$ and $g^{\prime}(0)$ decrease by about $70 \%$ and $25 \%$, respectively, as $M$ increases from 1 to 4 . This trend is opposite to that of the case $\mathrm{I}(b=1)$ where $f^{\prime \prime}(0)$ and $g^{\prime}(0)$ increase with $M$. The effect of $M$ on $-s^{\prime}(0)$ and the effect of $\lambda$ on $f^{\prime \prime}(0),-s^{\prime}(0)$ and $g(0)$ are qualitatively similar to those of case I $(b=1)$ and they increase with $M$ or $\lambda$. For $A=\lambda=1,-s^{\prime}(0)$ increases, by about $24 \%$ as $M$ increases from 1 to 4 .

\section{Conclusions}

The surface shear stresses in the $x$-and $y$-directions and the surface heat transfer increase with the increasing acceleration, magnetic and rotation parameters whether the magnetic field is fixed relative to the fluid or body except that the surface shear stress in $x$-direction and the surface heat transfer decrease with increasing magnetic parameter when the magnetic field is fixed relative to the body. For a certain value of the acceleration parameter the surface shear stress in $x$-direction vanishes. However, it does not imply flow separation since the flow is unsteady. Below this value of the acceleration parameter the surface shear stress in $x$-direction becomes negative and reverse flow occurs in the $x$-component of the velocity profiles.

\section{References}

1. Dorfiman LA Hydrodynamic resistance and heat loss of rotating solids (Trans. by N. Kemmer). Oliver and Boyd, Edingburgh, 1963

2. Kraith F (1968) Convection heat transfer in rotating system. Adv Heat Transfer 5: 130-251

3. Hoskin NE (1955) The laminar boundary layer on a rotating sphere. In 50 Jahre Grenzschichfforschung, Friedr. Vieweg u sohn, Braunschweig, 127-131

4. Siekmann I (1962) The calculation of the thermal laminar boundary layer on rotating system. Z Angew Math Phys 13: 468-482

5. Chao BT; Grief R (1974) Laminar forced convection over rotating bodies. J Heat Transfer 96: 463-466

6. Chao BT (1977) An analysis of forced convection over nonisothermal surfaces via universal functions, Recent Advances in Engineering Science. Proc 14th Ann Meet Soc Eng Sci, Lehigh Univ 471-483 
7. Lee MH; Jeng DR; De Witt KJ (1978) Laminar boundary layer transfer over rotating bodies in forced flow. J Heat Transfer 100: 496-502

8. Kumari M; Nath G (1982) Nonsimilar laminar incompressible boundary layer flow over a rotating sphere. Arch Mech 34: 147-164

9. Kumari M; Nath G (1982) Unsteady incompresible boundary layer flow over a rotating sphere. J Appl Mech 49: 234-236

10. Ece MC (1992) The initial boundary layer flow past a transplating and spinning rotational symmetric body. J Engng Math 26: 415-428

11. Ames WF Nonlinear Partial Differential Equations. Academic Press, New York, 1965

12. Blueman GW, Cole JD Similarity Method for Differential Equations. Springer Verlag, New York, 1974

13. Ovsiannikov LV Group Analysis of Differential Equations. Academic Press, New York 1982

14. Rogers C; Chadwick WF Backlung Transformations and their Applications. Academic Press, New York, 1983
15. Olver PJ Applications of Lie Groups to Differential Equations. Springer Verlag, Berlin, 1986

16. Ma PKH; Hui WH (1990) Similarity solutions of the twodimensional unsteady boundary layer equation. J Fluid Mech 216: 537-559

17. Sparrow EM; Eckert ERG; Minkowycz WJ (1962) Transpiration cooling in a magnetohydrodynamic stagnation-point flow, Appl Sci Res 11A: 125-147

18. Whittaker ET; Watson GN Modern Analysis. Cambridge University Press, London 1963

19. Takhar HS (1968) Free convection from a flat plate. J Fluid Mech 34: 81-89

20. Yang KT (1958) Unsteady laminar boundary layers in an incompressible stagnation flow. J Appl Mech 25: 421-427

21. Sears WR; Telionis DP (1975) Boundary layer separation in unsteady flow. SIAM J Appl Math 28: 215-235

22. Ingham DB (1984) Unsteady separation. J Comp Phys 53: 90-99 